\title{
ST-segment elevation myocardial infarction (STEMI) bypass: The importance of paramedics in an integrated STEMI system of care
}

\author{
Alix J. E. Carter, MD, MPH* \\ INTRODUCTION
}

The American Heart Association recommendation for care of an ST-segment elevation myocardial infarction (STEMI) is to provide percutaneous coronary intervention (PCI) as the first line of treatment, if it is possible, in a timely manner. ${ }^{1}$ This is defined as a reperfusion device within 90 minutes of first medical contact. This has evolved from "door to balloon"; the interval now begins with the first medical contact as the diagnostic (paramedic) electrocardiogram (ECG) and concludes with the device (e.g., wire, balloon) deployed. This recognizes the significant role of paramedics in an integrated STEMI system of care.

An optimized prehospital interval could involve transporting the patient directly to a PCI-capable facility, bypassing the closest emergency department (ED); this process is called STEMI bypass. Guidelines state that the treating paramedics should "be capable of managing complications associated with acute coronary syndrome." In many systems, primary care paramedics (PCP) are the first on scene because chest pain calls are high priority and the closest unit is dispatched. In some systems, there are no advanced care paramedics (ACP) at all. Can PCP safely care for and transport a STEMIbypass patient? Dispatching the more distant ACP, for example, would delay the time to aspirin, or first shock in the event that this evolves to a cardiac arrest. Requiring an ACP to transport a STEMI-bypass would also delay reperfusion. A pilot study conducted by Cantor et al. concluded that STEMI patients could be safely transported directly to PCI by paramedics without advanced care training. ${ }^{2}$ The paper published in this volume by Mitchell et al. echoes this recommendation and adds to our understanding of how to best use the skills and resources of paramedic services to safely minimize ischemic time. ${ }^{3}$

In the Mitchell paper, a PCP STEMI bypass program in a Canadian urban/suburban paramedic service is described (contact time with paramedics: 29 minutes, 40 seconds). ${ }^{3}$ This program began in 2006 and presents safety data for 2011-2016. Although a significant (59\%) proportion had clinically important events, complications were transient and often resolved with no therapy. Furthermore, a smaller proportion would be addressed differently by ACP (many were hypotension responding to fluid, which could be addressed by PCP where they can initiate intravenous [IV] and fluid bolus). They conclude that PCP can safely conduct a STEMI bypass.

This adds to the growing body of evidence on the role of paramedics in an integrated STEMI system of care, which includes two other Canadian papers recently published in CFEM. Bussieres et al. present a rural environment with longer transport times (>30 minutes to the PCI centre). ${ }^{4}$ Data on 1,114 patients over a period of 8 years reveal $18.5 \%$ major events and $12.2 \%$ minor for PCP STEMI bypass. Kwong et al. recently presented data from an urban paramedic service. ${ }^{5}$ One year of data included 232 PCP-only STEMI bypass, of which 21/232 (9.1\%) had indications for an ACP intervention. Eleven patients experienced cardiac arrest; 10 were successfully resuscitated ( 5 of these by PCPs).

What does this add to our understanding of a STEMI bypass? Evidence-based guidelines recommend direct to PCI, but these same guidelines say the treating paramedics should be capable of managing

From the *Department of Emergency Medicine, Division of EMS, Dalhousie University, Halifax, NS.

Correspondence to: Dr. Alix J. E. Carter, Dalhousie University, Department of Emergency Medicine, Division of EMS, 3018-1796 Summer St., Halifax, NS B3H 3A7; Email: alix.carter@novascotia.ca 
complications. What are the complications, how often do they happen, and who is capable of managing them? And what are the consequences of the alternative(s)? This series of papers helps us unpack these issues and make evidence-based decisions about system design for STEMI care.

Firstly, these three papers all address the question of what complications arise and how often. All three describe low incidence of events requiring intervention. These three authors also illustrate that few of these events would require an intervention outside of the PCP scope, particularly in a system that endorses PCP IV starts and fluid bolus. They present the option of an ACP intercept, possibly for a selected higher-risk population. Incidence of, and survival from, cardiac arrest was the same in ACP or PCP care. This aligns with prior work by Ryan et al. who studied a system without a PCP STEMI bypass and found that interventions requiring an ACP scope of practice occurred in fewer than $10 \%$ of 342 STEMI patients. The most frequent intervention was administration of morphine for chest pain. ${ }^{6}$

This provides mounting evidence that complications outside of the scope of a PCP are rather infrequent, and that waiting for an ACP, or transporting first to a nonPCI centre, may have limited added value. If time is muscle, what is the time cost of the alternatives to a PCP STEMI bypass? In a large urban setting, the median additional transport time of a PCP STEMI bypass compared to transport to the closest ED was 5.53 minutes (interquartile $[\mathrm{IQR}]=6.71$ ). In the event that it becomes necessary, the median additional time incurred by an ACP-rendezvous was low (7.49 v. 5.53 minutes). ${ }^{5}$ Further, transporting STEMI patients to the closest hospital with the need for subsequent interfacility transfer adds significantly more ischemic time. ${ }^{5}$ This is borne out in several studies, including Ross et al. who reported that, in 89 PCP STEMI cases transported to the nearest hospital, the ischemic time could have been decreased by an estimated 50 minutes with direct transport to a PCI centre while only incurring a 7 -minute increase in the transport interval. ${ }^{7}$

The literature provides evidence on other aspects of optimizing the prehospital interval and the paramedic skill set in an integrated STEMI system of care. In the Kwong paper, paramedics activate the PCI lab based on their own interpretation, guided by the automated computer interpretation. ${ }^{5}$ In Mitchell and Bussieres, paramedics transmit and a remote physician is involved in making the decision. ${ }^{3,4}$ Multiple studies document feasibility and accuracy of prehospital ECG by appropriately trained paramedics. ${ }^{8}$ Studies support a time savings from the first diagnostic ECG to device with prehospital acquisition of ECG. Multiple studies have supported the accuracy of paramedic interpretation. ${ }^{9}$ Removing the step of transmission has been shown to save time from first medical contact to balloon, and avoids the 1 in 5 chance of transmission failure. ${ }^{10}$ Transmission to, and direct communication with, interventional cardiology may help further reduce time to device. ${ }^{11}$

The Mitchell paper in this volume, supported by two recent $C F E M$ publications and a growing body of literature, endorses that a STEMI bypass shortens ischemic time; further, it should include regions that have few or no ACP. Treating STEMI with rapid access to PCI decreases ischemic time and improves outcomes. Optimizing paramedic scope of practice and enabling paramedic services to function to their full scope as part of an integrated STEMI system of care, including PCP STEMI bypass, would improve access to this service and improve outcomes from STEMI for more of the population.

\section{Keywords:}

acute coronary syndrome, EMS, paramedic, systems of care

Competing interests: None declared.

\section{REFERENCES}

1. O'Connor RE, Al Ali AS, Brady WJ, et al. Part 9: acute coronary syndromes: 2015 American Heart Association guidelines update for cardiopulmonary resuscitation and emergency cardiovascular care. Circulation 2015;132(18): S483-500, doi:10.1161/CIR.0000000000000263.

2. Cantor WJ, Hoogeveen P, Robert A, et al. Prehospital diagnosis and triage of ST-elevation myocardial infarction by paramedics without advanced care training. Am Heart 7 2012;164:201-6.

3. Mitchell S, Dionne R, Maloney J, et al. Safety and clinically important events in PCP-initiated STEMI bypass in Ottawa. CFEM 2018;20(6):865-73.

4. Bussières S, Bégin F, Leblanc P-A, et al. Clinical adverse events in prehospital patients with ST-elevation myocardial infarction transported to a percutaneous coronary intervention centre by basic life support paramedics in a rural region. CFEM 2018;20(6):857-64.

5. Kwong JL, Ross G, Turner L, et al. Evaluation of a primary care paramedic STEMI bypass guideline. CFEM 2018;20(6):850-56. 
6. Ryan D, Craig AM, Turner L, et al. Clinical events and treatment in prehospital patients with ST-segment elevation myocardial infarction. Prehosp Emerg Care 2013;17:181-6.

7. Ross G, Alsayed T, Turner L et al. Assessment of the safety and effectiveness of emergency department STEMI bypass by defibrillation-only emergency medical technicians/primary care paramedics. Prehosp Emerg Care 2015;19:191-201.

8. Ducas RA, Wassef AW, Jassal DS, et al. To transmit or not to transmit: how good are emergency medical personnel in detecting STEMI in patients with chest pain? Can 7 Cardiol 2012;28:432-7.

9. Cheskes S, Turner L, Foggett R, et al. Paramedic contact to balloon in less than 90 minutes: a successful strategy for
ST-segment elevation myocardial infarction bypass to primary percutaneous coronary intervention in a Canadian emergency medical system. Prehosp Emerg Care 2011;15:490-8.

10. Le May MR, Dionne R, Maloney J, Poirier P. The role of paramedics in a primary PCI program for ST-elevation myocardial infarction. Prog Cardiovasc Dis 2010;53:183-7.

11. Sejersten M, Sillesen M, Hansen PR, et al. Effect on treatment delay of prehospital teletransmission of 12-lead electrocardiogram to a cardiologist for immediate triage and direct referral of patients with ST-segment elevation acute myocardial infarction to primary percutaneous coronary intervention. Am 7 Cardiol 2008;101:941-6. 